\title{
Indicadores de qualidade utilizados nos serviços de enfermagem de hospitais de ensino
}

\author{
Quality indicators used in the nursing services of teaching hospitals
}

Indicadores de calidad utilizados en servicios de enfermería de hospitales de enseñanza

Mariana Angela Rossaneis ${ }^{1}$, Carmen Silvia Gabriel ${ }^{2}$, Maria do Carmo Fernandez Lourenço Haddad ${ }^{3}$, Marcia Regina Antonietto da Costa Melo ${ }^{4}$, Andrea Bernardes ${ }^{5}$

\footnotetext{
${ }^{1}$ Enfermeira, Mestre em Enfermagem Fundamental. Discente do Programa de Pós-Graduação em Enfermagem, nível Doutorado, da Universidade de Maringá.

Professora Auxiliar da Universidade de Londrina (UEL). Londrina, PR, Brasil. E-mail: marianarossaneis@gmail.com.

${ }^{2}$ Enfermeira, Doutora em Enfermagem em Saúde Publica. Professora Doutora da Escola de Enfermagem de Ribeirão Preto da Universidade de São Paulo

(EERP/USP). Ribeirão Preto, SP, Brasil. E-mail: cgabriel@eerp.usp.br.

${ }^{3}$ Enfermeira, Doutora em Enfermagem. Professora Associada da UEL. Londrina, PR, Brasil. E-mail: haddad@sercomtel.com.br.

${ }^{4}$ Enfermeira, Doutora em Enfermagem. Professora Doutora da EERP/USP. Ribeirão Preto, SP, Brasil. E-mail: mracmelo@eerp.usp.br.

${ }^{5}$ Enfermeira, Doutora em Enfermagem Fundamental. Professora Doutora da EERP/USP. Ribeirão Preto, SP, Brasil. E-mail: E-mail: andreab@eerp.usp.br.
}

\section{RESUMO}

Estudo descritivo de abordagem quantitativa cujo objetivo foi identificar a utilização de indicadores de qualidade da assistência de enfermagem em hospitais de ensino. A população foi composta por nove enfermeiros gestores de enfermagem de nove hospitais de ensino. A coleta de dados foi realizada por meio de um questionário eletrônico referente à caracterização dos enfermeiros e a utilização dos indicadores de qualidade nas instituições do estudo. Identificou-se que a maioria dos indicadores adotados pelo serviço são os indicadores gerais da instituição (taxas de infecção hospitalar, ocupação, mortalidade, etc.). Não se observou uma padronização dos indicadores adotados pelos hospitais, e estes também não realizavam a comparação dos seus resultados com os de outras instituições. A utilização de indicadores de qualidade já é prática nas instituições de saúde, porém ainda é necessário implementar estratégias de análise desses indicadores para que sejam passíveis de comparabilidade e reflitam os diferentes contextos da assistência à saúde.

Descritores: Indicadores de Qualidade em Assistência à Saúde; Qualidade da Assistência à Saúde; Avaliação em Saúde; Enfermagem.

\section{ABSTRACT}

Descriptive study of quantitative approach aimed at identifying the use of nursing care quality indicators in teaching hospitals. The population consisted of nine nursing care managers from nine teaching hospitals. Data collection was done through an electronic questionnaire on the characterization of the nurses and the use of quality indicators in these study institutions. It was identified that most of the indicators adopted by the service are the institution's general indicators (hospital infection rates, occupancy, mortality, etc.). A standardization of the indicators adopted by hospitals was not observed and they also did not perform a comparison of its results with other institutions. The use of quality indicators is already a practice in health institutions, but it is still necessary to implement strategies for analyzing these indicators so that they can be compared and reflect the different contexts of health care.

Descriptors: Quality Indicators, Health Care; Quality of Health Care; Health Evaluation; Nursing.

\section{RESUMEN}

Estudio descriptivo de abordaje cuantitativo, objetivando identificar la utilización de indicadores de calidad de atención de enfermería en hospitales de enseñanza. Población compuesta por nueve enfermeros jefes de enfermería de nueve hospitales de enseñanza. Datos recolectados mediante cuestionario informático, referido a la caracterización de los enfermeros y la utilización de indicadores de calidad en las instituciones estudiadas. Se identificó que la mayoría de los indicadores adoptados por el servicio son los generales de la institución (tasas de infección hospitalaria, ocupación, mortalidad, etc.). No se observó estandarización de los indicadores adoptados por los hospitales. Estos tampoco efectuaban comparación de sus resultados con los de otras instituciones. La utilización de indicadores de calidad ya es práctica habitual en las instituciones de salud; no obstante, aún es necesario implementar estrategias de análisis de tales indicadores para que sean pasibles de comparación y reflejen los diferentes contextos de la atención de salud.

Descriptores: Indicadores de Calidad de la Atención de Salud; Calidad de la Atención de Salud; Evaluación en Salud; Enfermería. 


\section{INTRODUÇÃO}

O alcance da excelência na qualidade da assistência é uma meta que vem sendo almejada por instituições de saúde, preocupadas em garantir por meio de suas ações o exercício profissional e de cidadania dos trabalhadores, bem como dos usuários dos serviços de saúde ${ }^{(1)}$.

Com as mudanças no padrão de comportamento da sociedade, o cidadão passou a exigir qualidade ao consumir produtos e serviços, deixando de agir de forma passiva e condescendente, exercendo uma pressão cada vez maior sobre a estrutura organizacional desses serviços $^{(2)}$. Além disso, o crescimento progressivo dos gastos no setor da saúde, principalmente nos hospitais, decorrente dos avanços tecnológicos e do aumento da complexidade assistencial, demandam dos gestores o enfrentamento de desafios constantes para viabilização destas instituições na oferta de serviços com qualidade ${ }^{(3)}$.

$\mathrm{Na}$ área da saúde, qualidade é definida como um conjunto de atributos que inclui nível de excelência profissional, uso eficiente de recursos, mínimo risco ao usuário/família, alto grau de satisfação dos usuários, considerando-se, essencialmente, os valores sociais existentes $^{(4)}$.

Embora o processo de trabalho nas instituições de saúde tenha passado por transformações que trouxeram avanços científicos e tecnológicos, ainda são vivenciados atendimentos desumanos, filas de espera, índices elevados de infecção hospitalar, gerenciamento ineficaz de custos, baixo nível de capacitação profissional e ausência de educação continuada / permanente, dimensionamento de pessoal inadequado, entre outras dificuldades. Essa situação clama por modelos de gestão adequados, que otimizem os recursos aplicados, melhorando a produtividade, a satisfação dos usuários e dos profissionais que atuam nos serviços de saúde ${ }^{(5)}$.

Inseridos neste contexto, os serviços de enfermagem enfrentam desafios para atender às demandas dos clientes internos e externos, a fim de alcançar a excelência da qualidade assistencial ${ }^{(6)}$. O enfermeiro é um dos profissionais que mais tem se envolvido na implantação e implementação da gestão da qualidade nas instituições hospitalares, uma vez que no desenvolvimento do seu processo de trabalho tem a oportunidade de interagir diretamente com o cliente e se aproximar do seu referencial para compreender seus anseios e expectativas, aprimorando a prática do cuidar com qualidade ${ }^{(5)}$.
A busca pela melhoria da qualidade tem sido incorporada na rotina desses profissionais e para tanto se faz necessário o controle da qualidade da assistência fundamentado em avaliações sistematizadas do cuidado, avalições essas que podem ser realizadas por meio da adoção indicadores que demonstrem sua evolução ao longo do tempo e permitam a comparação com referenciais internos e externos ${ }^{(7)}$.

Indicadores são medidas utilizadas para descrever uma situação existente, avaliar mudanças ou tendências durante um período de tempo e avaliar, em termos de qualidade e quantidade, as ações de saúde executadas ${ }^{(8)}$. Os indicadores de qualidade, desempenho e produtividade são importantes no planejamento e na tomada de decisão dos gestores dos serviços de saúde para o aprimoramento de processos e melhoria dos resultados da assistência. Obviamente, um valioso instrumento de gestão para a superação dos desafios impostos pela atualidade e para a busca da eficácia gerencial $^{(9)}$.

Sendo assim, considerou-se a realização desse estudo relevante, uma vez que a partir dos resultados obtidos sobre a prática da utilização de indicadores de qualidade na gerencia dos serviços de enfermagem de hospitais de ensino de grande porte, que assistem a maior parte dos usuários do Sistema Único de Saúde (SUS) na região onde o estudo foi realizado, acredita-se ser possível identificar os principais marcadores de qualidade utilizados por esses serviços e as necessidade de implementação de estratégias para avaliações subsequentes que permitam comparabilidade dos resultados das avaliações nos âmbitos interno e externos e subsidiam a elaboração e reajuste de metas e objetivos organizacionais na busca pela melhoria da qualidade da assistência prestada a população.

Mediante ao exposto, este estudo tem como objetivo identificar a utilização de indicadores de qualidade da assistência no gerenciamento de enfermagem de hospitais de ensino.

\section{MÉTODOS}

Trata-se de um estudo exploratório, descritivo, transversal de abordagem quantitativa, realizado em dez hospitais gerais de ensino de grande porte, localizados no Estado do Paraná, sendo que dessas, nove das instituições aceitaram participar da pesquisa. Vale destacar que o Brasil possui 171 hospitais de ensino credenciados pelo Ministério da Saúde e pelo Ministério da Educação ${ }^{(10)}$. 
A população do estudo foi constituída por nove enfermeiros que atuam na gerência dos serviços de enfermagem dos hospitais de ensino há pelo menos dois anos.

A coleta de dados procedeu-se por meio de questionário eletrônico elaborado no programa Google Drive ${ }^{\circledR}$ pelo pesquisador, tendo sido enviado o link de acesso ao instrumento por e-mail aos participantes do estudo. O questionário foi composto por questões fechadas, que correspondem à caracterização dos enfermeiros, segundo as variáveis: sexo, idade, anos de formação profissional, anos de experiência na assistência hospitalar, anos de experiência no gerenciamento dos serviços de enfermagem, anos que possuem vínculo empregatício com a instituição em estudo e a realização de pós-graduação; e relacionadas à utilização dos indicadores para avaliar a qualidade da assistência de enfermagem. $O$ instrumento foi previamente analisado e aprovado por três enfermeiras supervisoras de um hospital filantrópico de grande porte e por uma docente da área de administração em enfermagem, com fundamentos na experiência profissional de cada uma.

Procedeu-se a análise estatística descritiva dos dados no programa Microsoft Excel 2010. Este estudo foi submetido ao Comitê de Ética em Pesquisa da Universidade Estadual de Londrina e aprovado sob o parecer no 84178/2012 e CAAE 04053112.0.0000.5231.

\section{RESULTADOS}

Identificou-se na caracterização da população do estudo que a idade dos participantes variou entre 28 e
62 anos, com idade media de 45 anos. O tempo de formação variou de oito a 35 anos, com média de 21 anos. Em relação ao tempo que possuem vínculo empregatício com o hospital, houve uma variação de quatro a 26 anos, com média de 15 anos. Todos os participantes referiram que atuavam na assistência de enfermagem antes de assumirem o cargo de gerência.

Em relação ao tempo que possuem vínculo empregatício com o hospital, houve uma variação de quatro a 26 anos, com média de 15 anos. Todos os participantes referiram que atuavam na assistência de enfermagem antes de assumirem o cargo de gerência.

O tempo em que atuam na gerência dos serviços de enfermagem na instituição variou entre três e 19 anos, em média 11 anos. Todos os enfermeiros que participaram do estudo possuíam pós-graduação e sete especializaram-se na área de administração em saúde.

Quanto as instituições do estudo, no que refere-se as à participação em programas de avaliação e/ou certificação da qualidade da assistência, dois hospitais participam do Programa de Apoio e Qualificação de Hospitais Públicos e Filantrópicos do SUS Paraná (HOSPSUS), dois estão em processo de acreditação pela Organização Nacional de Acreditação (ONA) e cinco não participam de nenhum programa.

Importante destacar que uma das instituições respondeu que não adota indicadores de qualidade no gerenciamento dos serviços de enfermagem. Os indicadores utilizados pelas oito instituições estão descritos na Tabela 1.

Tabela 1: Indicadores de qualidade da assistência adotados no gerenciamento de enfermagem dos hospitais de ensino do Paraná, Brasil. 2012.

\begin{tabular}{|c|c|c|}
\hline Indicadores de qualidade da assistência de enfermagem & $\mathbf{n}=\mathbf{8}$ & $\%$ \\
\hline Incidência de não conformidade na administração de medicamentos & 5 & 62,5 \\
\hline Incidência de úlcera por pressão (UPP) & 6 & 75 \\
\hline Incidência de queda do paciente & 6 & 75 \\
\hline Incidência de flebite & 4 & 50 \\
\hline Incidência de obstrução de cateter venoso central & 2 & 25 \\
\hline Incidência de extubação não programada & 2 & 25 \\
\hline Perda de sonda nasogastroenteral & 1 & 12,5 \\
\hline Não conformidades nos registros de Enfermagem & 2 & 25 \\
\hline Taxa de infecção hospitalar & 8 & 100 \\
\hline Taxa de mortalidade hospitalar & 7 & 87,5 \\
\hline Taxa de ocupação hospitalar & 8 & 100 \\
\hline Média de permanência hospitalar & 7 & 87,5 \\
\hline Horas de treinamento de profissionais de Enfermagem & 5 & 62,5 \\
\hline Taxa de acidente de trabalho de profissionais de enfermagem & 6 & 75 \\
\hline Taxa de rotatividade de enfermagem & 3 & 37,5 \\
\hline Distribuição enfermeiros/leito & 6 & 75 \\
\hline Distribuição técnicos e auxiliares de enfermagem/leito & 7 & 87,5 \\
\hline Taxa de absenteísmo de enfermagem & 6 & 75 \\
\hline Satisfação do cliente com a enfermagem & 5 & 62,5 \\
\hline
\end{tabular}


Os indicadores adotados pela maioria dos hospitais do estudo para avaliar os serviços de enfermagem foram os indicadores gerais da instituição: taxa de infecção hospitalar, taxa de ocupação hospitalar e média de permanência hospitalar.

Em relação aos indicadores da assistência direta ao paciente, os mais utilizados foram à incidência de não conformidade na administração de medicamentos, incidência de UPP, incidência de queda do paciente e a taxa de acidente de trabalho de profissionais de enfermagem.

Ainda, os indicadores horas de treinamento de profissionais de enfermagem, distribuição enfermeiros/leito, distribuição técnicos e auxiliares de enfermagem/leito e taxa de absenteísmo de enfermagem, relacionados principalmente com gerenciamento de recursos humanos, também são utilizados pela maior parte das instituições, assim como o indicador satisfação do cliente com a enfermagem.

Quanto à participação em banco de dados para a comparação de indicadores com outras instituições, nenhuma das instituições referiu realizar essa prática.

Todos os enfermeiros referiram que os dados dos resultados dos indicadores de qualidade são divulgados para a equipe de enfermagem e, em 55,5\% das instituições, os dados são discutidos com a equipe de enfermagem. Em oito instituições os resultados das avaliações são utilizados na elaboração do programa de capacitação/educação da equipe.

\section{DISCUSSÃO}

A caracterização dos enfermeiros gestores dos serviços de enfermagem demonstrou que a maioria era do sexo feminino e a média de idade foi de 43 anos. Um dos diferenciais do gerenciamento na área da enfermagem é a presença de mulheres assumindo cargos de chefia, conforme identificado no estudo. A tendência da ocupação de cargos gerenciais na área de saúde por mulheres também foi identificada em estudo que analisou o perfil gerencial dos enfermeiros de hospitais acreditados pela ONA e pela Joint Commission on Accreditation of Healthcare Organizations do Município de São Paulo-SP(10).

Assim como revelado nesse estudo, uma pesquisa realizada em um hospital público federal de ensino também identificou que os mais jovens não têm ocupado cargos gerenciais nas instituições. Este fato sugere que o setor público elege normas gerenciais mais tradicionais, em que a construção de carreiras ao longo da vida profissional é levada mais em consideração quando na escolha de gerentes ${ }^{(11)}$.

A maioria dos participantes do estudo $(55,5 \%)$ possui entre 21 a 30 anos de formados. Estudo que analisou o perfil necessário para o cargo de gerente de enfermagem identificou que o tempo de graduação mínima do enfermeiro deveria ser de cinco anos. Estimase que o fato de possuir maior tempo de formado e experiência profissional dá a esse profissional maior conhecimento, maturidade, experiência e segurança para exercer cargos de gerência ${ }^{(10)}$.

Todos os entrevistados possuíam certificação lato sensu e sete haviam realizado especializações relacionadas à área de gestão em saúde. Quatro dos participantes possuíam cursos de pós-graduação stricto sensu. Esses resultados podem estar relacionados ao fato dos enfermeiros atuarem em hospitais de ensino vinculados a instituições de ensino superior.

O enfermeiro ao assumir cargos de chefia nos serviços de saúde necessita adquirir, desenvolver e aperfeiçoar competências gerenciais. $\mathrm{Na}$ maioria das vezes, busca complementar e qualificar a formação obtida na graduação por meio de cursos latu e stricto senso. A realização de curso de pós-graduação demostra o compromisso dos enfermeiros com a qualidade de seu desempenho profissional, o que é favorável para o alcance de bons resultados na sua atuação e melhoria dos indicadores de qualidade do serviço ${ }^{(12)}$.

Dentre os que participam de programas de certificação de qualidade $(44,4 \%)$, dois fazem parte do HOSPSUS e dois estão em processo de acreditação por instituições acreditadoras credenciadas pela ONA.

O HOSPSUS é um programa do governo do Estado do Paraná que tem como objetivo melhorar a efetividade e a qualidade da assistência hospitalar para o atendimento das necessidades e demandas da população. Atualmente, o programa conta com 49 hospitais públicos e filantrópicos que são retaguarda para a Rede Mãe Paranaense e a Rede Estadual de Urgência e Emergência(13).

Este programa fundamenta-se no estabelecimento de compromissos entre o hospital e a Secretaria de Saúde do Estado. Esses compromissos são concretizados mediante contrato/convênio no qual a Secretaria repassa recursos financeiros e os hospitais se comprometem, em contrapartida, a promover a melhoria da qualidade da assistência com o cumprimento de metas preestabelecidas pelo contrato/convênio ${ }^{(13)}$. 
O processo de avaliação é realizado por meio de indicadores de desempenho agrupados nas seguintes áreas temáticas: gestão, estrutura, processo e resultado. Esse método de avaliação fundamenta-se na trilogia de Donabedian $^{(14)}$ em que a estrutura corresponde aos recursos utilizados, o processo às atividades relativas à organização da assistência, à produtividade, à produção e utilização de recursos, nos seus aspectos quantitativos e qualitativos e o resultado à efetividade do cuidado, entendida como o grau com que a assistência hospitalar atinge o objetivo de promover a saúde dos indivíduos. A área de gestão foi incluída devido ao caráter do programa de promoção da melhoria gerencial(13-14).

Essa iniciativa do Governo do Estado do Paraná representa um avanço no método de analise do desempenho das instituições de saúde, pois a avaliação dos serviços por meio de indicadores permite identificar objetivos, obter informações reais, conhecer resultados e comparar parâmetros de desempenho entre as instituições.

Sendo assim, as instituições que participam do programa HOSPSUS buscam na avaliação dos seus serviços a identificação das fragilidades nos seus processos e o desenvolvimento de estratégias para a melhoria de desempenho e qualidade assistencial. Outra metodologia de avaliação identificada entre os hospitais de estudo foi a participação em programas de acreditação hospitalar realizados por instituições credenciadas pela ONA.

Apesar de também ser um método de avaliação, o objetivo das instituições que se submetem a acreditação são diferentes dos hospitais que participam do HOSPSUS. Os hospitais em processo de avaliação por instituições credenciadas pela ONA buscam na acreditação a certificação do padrão de qualidade que acreditam já possuir nos serviços prestados.

No método de acreditação realizado pela ONA, após a avaliação e constatação que os serviços prestados pela instituição alcançam os padrões de qualidade, a agência acreditadora concede um selo de certificação. Para manter o selo de certificação, a instituição deve no mínimo manter a qualidade ou melhorá-la, uma vez que a avaliação da qualidade é um processo dinâmico, ininterrupto e de exaustiva busca de falhas nas rotinas e procedimentos, que devem ser periodicamente revisados, atualizados e difundidos ${ }^{(15)}$.

No Brasil, 167 hospitais são acreditados pela ONA, sendo que a $73,3 \%$ estão localizados na região sudeste, principalmente nos estados de São Paulo e Minas Gerais.
$\mathrm{Na}$ região sul, encontram-se $11,9 \%$ dos hospitais acreditados e 12 dessas instituições estão localizados no Paraná(16).

Tanto instituições públicas como privadas de saúde estão buscando a certificação, como um mecanismo de qualidade, com base nas sugestões de que esse processo é benéfico, mesmo quando seus resultados não são tangíveis ${ }^{(17)}$.

Independente do programa de avaliação do qual os hospitais participam, pode-se identificar uma cultura de valorização das informações com vistas a discutir e implementar melhorias assistenciais nas instituição do estudo, uma vez que os gestores desses serviços utilizam dados gerenciais $e$ indicadores para o acompanhamento de resultados e metas.

A melhoria contínua da qualidade assistencial só é realizada com a identificação dos fatores intervenientes no processo de trabalho da organização e exige a implementação de ações e a elaboração de instrumentos que possibilitem avaliar de maneira sistemática os níveis de qualidade dos cuidados prestados ${ }^{(7)}$.

A avaliação é parte fundamental no planejamento e na gestão dos serviços de saúde de saúde. O processo de avaliação realizado de forma efetiva possibilita a reordenação das práticas assistenciais, de forma a contemplar as necessidades dos usuários e com maior racionalidade no uso dos recursos disponíveis.

Apesar do Manual Brasileiro de Acreditação, instituído pela ONA, e o Manual do HOSPSUS não identificarem especificamente quais indicadores devem ser utilizados para avaliar os serviços de enfermagem, ambos definem claramente que todos os serviços envolvidos no cuidado ao paciente, incluindo a Enfermagem, devem ser avaliados por meio de indicadores para analisar a estrutura, o processo e os resultados da assistência prestada ${ }^{(13,16)}$.

O emprego de indicadores possibilita aos gestores dos serviços de saúde monitorar e avaliar os eventos que acometem os usuários, os trabalhadores e as organizações, apontando, como consequência, se os processos e os resultados organizacionais vêm atendendo às necessidades e expectativas dos usuários $^{(1)}$.

Quanto aos indicadores utilizados no gerenciamento dos serviços de enfermagem, os que mais se destacaram foram os relacionados ao resultado da assistência: taxa de infecção hospitalar e taxa de ocupação hospitalar com 100,0\%; taxa de mortalidade e média de permanência hospitalar, ambos utilizados em $87,5 \%$ das instituições. 
Esses indicadores, geralmente, são analisados pela direção do hospital, fora do rol de indicadores específicos da enfermagem. Eles são utilizados na avaliação dos resultados da assistência em saúde à medida que são influenciados por índices de resolutividade alcançados pela equipe assistencial ${ }^{(18)}$.

Salienta-se que a enfermagem tem interferência direta nos resultados da assistência prestada ao paciente e por isso também deve utilizar esses indicadores no gerenciamento dos seus serviços.

Os indicadores mais utilizados nas instituições do estudo, relacionados à assistência de enfermagem direta ao paciente foram: incidência de úlcera por pressão, incidência de queda do paciente, incidência de não conformidade na administração de medicamentos e taxa de acidente de trabalho de profissionais de enfermagem.

Esses indicadores fazem referência aos processos assistenciais específicos da enfermagem, os quais estão estreitamente relacionados às tarefas desenvolvidas diariamente pelos profissionais e são capazes de retratar a qualidade desses processos. A maioria dos indicadores específicos para avaliar a qualidade do cuidado da enfermagem está vinculada a analise da estrutura e do processo. Isso ocorre por possibilitarem dados mais objetivos e concretos. Sendo assim, no gerenciamento de enfermagem os indicadores mais utilizados são aqueles que se relacionam aos cuidados físicos $^{(18)}$

Em relação aos indicadores de gestão de pessoas os mais utilizados foram à distribuição de técnicos e auxiliares de enfermagem/leito, distribuição de enfermeiros/leito, taxa de absenteísmo de enfermagem e horas de treinamento de profissionais de enfermagem.

No âmbito da gerência e, mais especificamente, no que diz respeito à gestão de pessoas em saúde e em enfermagem, o uso de indicadores não tem sido adotado e chama a atenção o seu reduzido número( ${ }^{(8)}$.

Observou-se que além dos indicadores de dimensionamento de pessoal, a maioria das instituições utiliza apenas o indicador horas de treinamento de profissionais de enfermagem no gerenciamento dos seus serviços.

Devido à complexidade e à diversidade dos fatores que influenciam o gerenciamento do trabalho de enfermagem, apenas horas de treinamento não avaliam essa função administrativa sob a responsabilidade do enfermeiro. A utilização de indicadores para a avaliação da gestão de pessoas permite detectar a eficiência e a eficácia das ações gerenciais e a partir de avaliação dos seus resultados, aprimorar os processos de trabalho. Faz-se necessário a construção e validação de outros indicadores sensíveis para avaliar a gestão de pessoas nos serviços de enfermagem ${ }^{(8)}$.

Acredita-se que $\mathrm{o}$ indicador taxa de rotatividade tenha sido pouco mencionado porque a maioria das instituições é administrada pelo Governo do Estado do Paraná. Sendo assim, nessas organizações a forma de contratação é por meio de concurso público que não ocorre frequentemente e os trabalhadores permanecem por mais tempo na instituição devido às melhores condições salariais.

Ainda, segundo os entrevistados, o indicador satisfação do cliente com a enfermagem é utilizado em $62,5 \%$ dos serviços. Esse indicador é um dos mais importantes quando se discute avaliação dos serviços de saúde, uma vez que permite avaliar se as necessidades dos usuários, foco principal das organizações hospitalares, foram atingidas.

A escuta da opinião de quem recebe a assistência é um elemento importante na avaliação dos serviços de saúde, bem como da forma que o sistema se organiza para viabilizar o atendimento. A incorporação do usuário na avaliação tem sido valorizada não apenas por constituir-se um indicador sensível da qualidade do serviço prestado, mas por estar potencialmente relacionada à maior adequação no uso do serviço ${ }^{(19)}$.

Os indicadores incidência de flebite, incidência de obstrução de cateter venoso central, incidência de extubação não programada, perda de sonda nasogastroenteral e não conformidades nos registros de enfermagem foram os indicadores identificados como os menos utilizados nas instituições que participaram do estudo.

O fato das instituições participantes do estudo não fazerem parte de programas de qualidade específicos da enfermagem não há uma padronização dos indicadores utilizados para avaliação dos serviços.

O estabelecimento de um rol de indicadores comum a todas as instituições permitiria a comparação periódica dos resultados da assistência de enfermagem, contribuindo assim para a identificação das falhas na produção do cuidado e estratégias de melhoria da qualidade.

A utilização de indicadores traz como benefícios o monitoramento da qualidade da assistência de enfermagem que está sendo oferecida ao usuário, bem como permite a comparação dos resultados das avaliações entre hospitais e, em um mesmo hospital ao 
longo do tempo, na busca dos fatores intervenientes no processo de cuidar em saúde.

Estudo realizado em instituições participantes ao Programa Controle de Qualidade Hospitalar de São Paulo identificou que o fato dos hospitais realizarem o monitoramento dos seus serviços por meio de indicadores pré-definidos e participarem de um banco de dados para a comparação dos resultados desses indicadores proporciona a avaliação regular dos serviços e a identificação dos fatores intervenientes específicos da qualidade da enfermagem. Sendo assim, favorece o estabelecimento de estratégias de intervenção em cada etapa do processo de trabalho(7).

As instituições de saúde vêm utilizando e desenvolvendo indicadores para o acompanhamento de resultados e metas, e há consentimento sobre a importância dessa ferramenta para avaliar a qualidade e desempenho da enfermagem ${ }^{(6-7)}$.

Agir gerencialmente requer tomada de decisão e planejamento que devem ser fundamentados nos resultados de avaliações realizadas por meio de indicadores, visando à formulação de estratégias de adequação dos serviços aos padrões de qualidade existentes.

\section{CONCLUSÃO}

\section{REFERÊNCIAS}

1. Tronchin DMR, Meleiro MM, Takahasshi RT. A qualidade e a avaliação dos serviços de saúde e de enfermagem. In: Kurcgant P, Tronchin DMR, Peres HHC, Massarollo MCKB, Fernandes MFP, Ciampone MHT et al. Gerenciamento em enfermagem. 2. ed. Rio de Janeiro (RJ): Guanabara Koogan; 2010. p.77-107.

2. Barbosa LR, Melo MRAC. Relações entre qualidade da assistência de enfermagem: revisão integrativa da literatura. Rev Bras Enferm. 2008 Mai-Jun; 61(2): 366-70.

3. Bonacim CAG, Araujo AMP. Avaliação de desempenho econômico-financeiro dos serviços de saúde: os reflexos das políticas operacionais no setor hospitalar. Ciênc saúde coletiva. 2011; 16(supl.1): 1055-69.

4. Organização Mundial de Saúde. Avaliação dos Programas de Saúde: normas fundamentais para sua aplicação no processo de gestação para o desenvolvimento nacional de saúde. Genebra: Organização Mundial de Saúde; 1981.

5. Rocha ESB, Trevizan MA. Gerenciamento da qualidade em um serviço de enfermagem hospitalar. Rev Latino-Am Enfermagem [online]. 2009 [cited 2013 jan 17]; 17(2). Available from: http://www.scielo.br/pdf/rlae/v17n2/pt_16.pdf

6. Simões e Silva C, Gabriel CS, Bernardes A, Évora YDM. Opinião do enfermeiro sobre indicadores que avaliam a qualidade na assistência de enfermagem. Rev Gaúcha Enferm. 2009 Jun; 30(2):263-71.

7. Gabriel CS, Melo MRAC, Rocha FLR, Bernardes A, Miguelaci T, Silva MLP. Utilização de indicadores de desempenho em serviço de enfermagem de hospital público. Rev Latino-Am Enfermagem [online]. 2011 [cited 2013 jan 17]; 19(5). Available from:
Identificou-se nesse estudo que a enfermagem vem ocupando cada vez mais espaço no quadro gerencial das instituições de saúde devido às habilidades desenvolvidas em liderança de equipes e gerenciamento de recursos humanos, materiais e físicos.

A maioria dos indicadores utilizados no gerenciamento dos serviços de enfermagem são os indicadores gerais da instituição e indicadores específicos da enfermagem relacionados à assistência direta aos pacientes. Contudo, não há uma padronização nos indicadores utilizados entre as instituições do estudo, sendo que cada uma estabelece um rol de indicadores para seguir.

Conclui-se com essa pesquisa que a utilização de indicadores de qualidade já é prática nas instituições de saúde, porém ainda é necessário implementar estratégias de análise desses indicadores para que sejam passíveis de comparabilidade e reflitam os diferentes contextos da assistência a saúde prestada à população a fim de subsidiar programas e politicas publicas de melhoria da qualidade assistencial.

Considera-se como limitação desse estudo o número de hospitais de ensino que fizeram parte do estudo, entretanto, apesar de limitada, a população do estudo representa uma parcela significativa de instituições que prestam atendimento ao Sistema Único de Saúde no Estado do Paraná.

http://www.scielo.br/scielo.php?pid=S010411692011000500024 \&script=sci_arttext\&tIng=pt

8. Vieira APM, Kurcgant P. Indicadores de qualidade no gerenciamento de recursos humanos em enfermagem: elementos constitutivos segundo percepção de enfermeiro. Acta paul enferm. 2010; 23(1): 11-15.

9. Brasil. Ministério da Saúde. Portal da Saúde [online]. 2011 [cited em 2013 jan 17]. Available from:

http://portal.saude.gov.br/portal/saude/Gestor/visualizar_texto.c fm?idtxt=36281\&janela $=1$

10. Furukawa PO, Cunha ICKO. Perfil e competências de gerentes de enfermagem de hospitais acreditados. Rev Latino-Am Enfermagem [online]. 2011 [cited 2013 jan 17]; 19(1). Available from: http://www.scielo.br/pdf/rlae/v19n1/pt_15.pdf 11. Nóbrega MFB, Matos MG, Silva LMS, Jorge MSB. Perfil gerencial de enfermeiros que atuam em um hospital público federal de ensino. Rev enferm UERJ. 2008 Jul-Set;16(3): 333-8. 12. Santos I, Castro CB. Características pessoais e profissionais de enfermeiros com funções administrativas atuantes em um hospital universitário. Rev Esc Enferm USP. 2010; 44(1): 154-60. 13. Paraná. Governo do Estado. Secretaria de Saúde. Programa de apoio e qualificação de hospitais públicos e filantrópicos do sistema único de saúde do Paraná (HOSPSUS). Curitiba (PR): Governo do Estado do Paraná, 2011. p.74.

14. Donabedian A. The definition of quality and approach to its assessment. Ann Harbor (Michigan): Health Administration Press, 1980.

15. Feldman LB. Padrões e critérios para avaliar o serviço de enfermagem. In: D'Innocenzo M, Feldman LB, Fazenda, NRR, 
Helito RAB, Ruthes RA. Indicadores, auditorias, certificações.

2.ed. São Paulo (SP): Martinari; 2010. P.49-67.

16. Organização Nacional de Acreditação. Certificações

concedidas pela Organização Nacional de Acreditação [online].

2013 [cited 2013 jan 17]. Available from:

https://www.ona.org.br/Inicial. Acesso em: 12 jan. 2013.

17. Fortes MTR, Baptista TWF. Accreditation: tool or policy for health systems organizations? Acta paul enferm. 2012; 25(4): 626-31.

18. Caldana G, Gabriel CS, Bernardes A, Évora YDM. Indicadores de desempenho em serviço de enfermagem hospitalar: revisão integrativa. Rev Rene. 2011 Jan-mar; 12(1): 189-97.

19. Moimaz SAS, Marques JAM, Saliba O, Garbin CAS, Zina LG, Saliba NA. Satisfação e percepção do usuário do SUS sobre o serviço público de saúde. Physis. 2010; 20(4): 1419-40.

Artigo recebido em 15/02/2013.

Aprovado para publicação em 30/05/2014.

Artigo publicado em 31/12/2014. 